\title{
La gestión ambiental en la competitividad de las PYMES del Ecuador
}

\section{Environmental management in the competitiveness of PYMES in Ecuador}

\author{
Dra. Andreína Inés González Ordóñez \\ MBA. Tania Patricia Alaña Castillo \\ M.Sc. Sandy Jahaira Gonzaga Añazco \\ Universidad Metropolitana, Ecuador
}

Autor para correspondencia: aigonzalez@umet.edu.ec, talana@umet.edu.ec, sgonzaga@umet.edu.ec

Fecha de recepción: 04 de Agosto de 2017 - Fecha de aceptación: 10 de Agosto de 2017

Resumen: La gestión ambiental y la competitividad poseen relación directa, por ello la necesidad de implementar sistemas de gestión ambiental en las pequeñas y medianas empresas (pymes) como ventaja competitiva que les permita mejorar su posición en el mercado e ingresar a mercados más exigentes y como herramienta para controlar y prevenir los impactos negativos de sus actividades, productos y servicios sobre el ambiente. En el presente trabajo se analizó desde el punto de vista teórico la incorporación de la gestión ambiental en las pymes del Ecuador y su contribución al incremento de la competitividad de estas empresas y a la disminución de los problemas ambientales que puedan generar. Para ello se realizó una investigación documental a partir de artículos de revistas, trabajos de grado y literatura relacionada con el tema estudiado. Los resultados obtenidos permiten evidenciar que las grandes empresas son las que tienen un mayor porcentaje de la certificación ISO 14001 por sus condiciones financieras, lo que no ocurre con las pymes que las consideran complejas, costosas y difíciles de mantener. Existen pymes que cuentan con un sistema de gestión ambiental informal, lo cual les permite mejorar su desempeño, asumir su responsabilidad ante la contaminación causada en el ambiente y tomar los correctivos necesarios. Entre las principales actividades ambientales desarrolladas por las pymes se encuentran: ahorro de energía, reciclaje, ahorro de agua, reproceso de materiales, tratamiento de residuos y monitoreo de impactos. Es necesario fortalecer a las pymes en materia de gestión ambiental.

Palabras clave: pymes; gestión ambiental; competitividad

Abstract: The environmental management and competitiveness have a direct relationship, therefore the need to implement environmental management systems in small and medium sized enterprises (pymes) as a competitive advantage that allows them to improve their position in the market and enter more demanding markets and as a tool to control and prevent impacts negative effects of their activities, products and services on the environment. In the present work, the incorporation of environmental management in pymes in Ecuador and their contribution to increasing the competitiveness of these companies and the reduction of the environmental problems that they generate are analyzed from the theoretical point of view. For this purpose, a documentary research was done based on journal articles, degree papers and literature related to the subject studied. The results show that large companies have the highest percentage of ISO 14001 certification due to their financial conditions, which is not the case with pymes that consider 
them complex, expensive and difficult to maintain. There are pymes that have an informal environmental management system, which allows them to improve their performance, take responsibility for pollution caused in the environment and take corrective action. Among the main environmental activities developed by pymes are: energy saving, recycling, water saving, material reprocessing, waste treatment and impact monitoring. It is necessary to strengthen pymes in environmental management.

Key words: pymes; environmental management; competitiveness

\section{Introducción}

Las pequeñas y medianas empresa (pymes) juegan un papel de gran importancia dentro del desarrollo de toda economía debido a su relación e incidencia en la generación de empleo y crecimiento económico, y de manera general a nivel mundial, representan en promedio el $80 \%$ de los negocios de una economía. En este sentido, la importancia de este sector de la economía es trascendental para la generación de empleos, desarrollo de la producción, y el manejo sostenible de la economía (Veintimilla, 2014).

En la actualidad las pymes deben desarrollar sus actividades sin causar impactos ambientales y cumplir las leyes ambientales, por lo que es necesario desarrollar una gestión ambiental en forma preventiva, lo que contribuirá a que estas empresas logren un mejor desempeño ambiental y mejorar sus procesos y productos, lo que se verá reflejado en un aumento de la productividad y en una disminución de la contaminación.

Es importante resaltar que la gestión ambiental está conformada por el conjunto de políticas y actividades que las empresas desarrollan con la finalidad de proteger y conservar los elementos que conforman el sistema ambiental mediante un manejo integral de los mismos que conduzcan a la prevención y mitigación de los problemas de carácter ambiental (González, 2017).

Van Hoof (2005) resalta que para la mayoría de las pymes la gestión ambiental no se constituye en un elemento relevante y prioritario para su gestión. Esta baja prioridad se debe a un conjunto de factores como el alto nivel de informalidad, la poca exigencia de sus mercados, principalmente locales, y la baja capacidad de las autoridades ambientales para ejercer control y cumplimiento de la normatividad ambiental.

Por su parte, Bercovich \& López (2005) mencionan, entre las causas de las insuficiencias en la gestión ambiental de las pymes, las siguientes: la falta de información y desconocimiento de la normativa vigente y los estándares que les corresponde cumplir, sus requisitos y plazos; las dificultades de tipo económico ya que las empresas son reacias a enfrentar los costos que supone todo el proceso de auditoría ambiental, así como la identificación de tecnologías que deben ser incorporadas; se presentan dificultades de acceso a financiamiento, en particular a líneas específicas para gestión ambiental o producción limpia, aunado a que los bancos, en general, no poseen ni líneas específicas ni personal capacitado para entender proyectos en el área ambiental, además, las firmas necesitan apoyo para preparar y presentar solicitudes de financiamiento de proyectos ambientales. 
En tal sentido, Rodríguez Becerra \& Van Hoof (s.f.) expresan que la gestión ambiental empresarial en las pymes se puede convertir en una de las armas para enfrentar la apertura de los mercados, siempre y cuando los diferentes actores del sector empresarial y ambiental logren orientarse hacia nichos potenciales, para ello se requiere adaptar una nueva gestión que fortalezca la coordinación entre los mismos y salir del enfoque tradicional de una gestión ambiental aislada, integrándola a la competitividad de las empresas y región en su conjunto, ya que como lo indican Ojeda \& Mul (2015) existe una relación lineal positiva entre la gestión ambiental y la competitividad.

Saavedra, Milla \& Tapia (2013) citando a Solleiro \& Castañón (2005), señalan que la competitividad es la capacidad de una organización para mantener o incrementar su participación en el mercado basada en nuevas estrategias empresariales, en un sostenido crecimiento de la productividad, en la capacidad inter empresarial para participar en negociaciones con diferentes instituciones y otras compañías dentro de su ambiente, en la existencia de un ambiente competitivo determinado por el sector y el mercado de los consumidores y en políticas introducidas por los gobiernos nacionales y alianzas económicas regionales.

La gestión ambiental y la competitividad poseen relación directa, por ello la necesidad de implementar sistemas de gestión ambiental en las pymes donde la alta dirección asuma su compromiso, además es necesario la participación total de los trabajadores para hacer frente y manejar los problemas ambientales así como plantear los objetivos ambientales; el entrenamiento y la formación de habilidades en los trabajadores para cumplir sus responsabilidades ambientales y lograr objetivos ambientales, y por último el uso de indicadores ambientales para medir el desempeño ambiental de las empresas, de tal manera que se minimice el impacto negativo sobre el ambiente (González, 2017).

En el presente artículo se analiza la incorporación de la gestión ambiental en las pymes del Ecuador y su contribución al incremento la competitividad de estas empresas y a la disminución de los problemas ambientales que puedan generar.

Para cumplir con los objetivos propuestos, la investigación fue desarrollada a nivel descriptivo. Se realizó una investigación documental que permitió a través del método deductivo y el uso de las técnicas del análisis de contenido y la contratación de documentos y bibliografía relacionada con la gestión ambiental y la competitividad en las pymes, generar los aportes de la presente investigación.

\section{Desarrollo}

A continuación se presenta un análisis de las pymes del Ecuador y las actividades de gestión ambiental que han implementado las mismas para mejorar su competitividad y proteger el ambiente.

\section{Las pymes en el Ecuador}

Las pymes pueden definirse como entidades independientes dedicadas a la producción de bienes o a la prestación de servicios para satisfacer diferentes necesidades del mercado (Arévalo \& Morocho, 2016). 
Una organización pyme, es un ente productivo o de servicios, que genera empleo y productividad al país y permite abastecer la demanda de productos y servicios de empresas nacionales, multinacionales en industrias que mueven al Ecuador (Holguín \& Navas, 2015). Para de determinar si una empresa está dentro del grupo de las pymes, se deben tomar en cuenta algunos parámetros como son: personal que labora en la empresa, ventas netas al año e impuestos internos y valor de activos productivos netos de amortizaciones.

Estos autores señalan que las pymes en el Ecuador se encuentran en particular en la producción de bienes y servicios, siendo la base del desarrollo social del país tanto produciendo, demandando y comprando productos o añadiendo valor agregado, por lo que se constituyen en un actor fundamental en la generación de riqueza y empleo.

León (2015) señala que según el tamaño, las empresas pueden clasificarse en cuatro categorías: microempresas, pequeñas empresas, medianas empresas y grandes empresas. Para determinar el tamaño de las empresas, se toma en cuenta el nivel de valor bruto de las ventas anuales y número de personal ocupado en la actividad, según el artículo 3 de la Decisión 702 de la Comisión de la Comunidad Andina (2008), citado por León (2015), la clasificación es la siguiente:

Tabla 1. Clasificación de las empresas según su tamaño

\begin{tabular}{ccc}
\hline Tipo de empresa & Personal ocupado & Valor Bruto de las ventas anuales (US\$) \\
\hline Microempresa & 1 a 9 & $\leq 100.000$ \\
Pequeña empresa & 10 a 49 & $100.001-1.000 .000$ \\
Mediana empresa (A) & 50 a 99 & $1.000 .0001-2.000 .000$ \\
Mediana empresa (B) & 100 a 199 & $2.000 .001-5.000 .000$ \\
Grande empresa & Más de 200 & Más de 5.000.000 \\
\hline
\end{tabular}

Fuente: León (2015)

El conjunto de las microempresas, pequeñas y medianas empresas se conoce como MIPYME y el conjunto de pequeñas y medianas empresas se conoce como PYME. En el Ecuador el 89,6\% de las empresas son microempresas, el 8,2\% pequeñas, el 1,7\% medianas y el 0,5\% son grandes de un total de 704.556 empresas (INEC, 2014, citado por León, 2015).

Para Carrión, Zula \& Castillo (2016), las pequeñas y medianas empresas (pyme), son formas de organización empresarial que responden por la producción de una buena parte de los bienes y servicios en la sociedad contemporánea. El Servicio de Rentas Internas de Ecuador (SRI, 2015; citado por Carrión, Zula \& Castillo, 2016) define a las pyme como el conjunto de pequeñas y medianas empresas que de acuerdo con su volumen de ventas, capital social, cantidad de trabajadores, y su nivel de producción o activos presentan características propias de este tipo de entidades económicas.

En el Ecuador las pequeñas y medianas empresas se han formado realizando diferentes tipos de actividades económicas entre las que se destaca las siguientes: comercio al por mayor y al por menor; agricultura, silvicultura y pesca; industrias manufactureras; construcción; transporte, almacenamiento, y comunicaciones; bienes inmuebles y servicios prestados a las empresas y servicios comunales, sociales y personales (Servicio de Rentas Internas, 2010; citado por Alcocer \& Díaz, 2016). 
Barrera (2001), citado por León (2015) destaca las siguientes características positivas de este sector productivo: son factores claves para generar riqueza y empleo; es el factor clave para dinamizar la economía de regiones y provincias deprimidas, por ende dinamizará la economía ambiental en el país; mantiene alta capacidad para proveer bienes y servicios a la gran industria (subcontratación), por lo que se pueden formar cadenas de producción cerradas con las grandes empresas; es flexible para asociarse y enfrentar exigencias ambientales del mercado; movilidad operativa; gestión de localización e implementación rápida de procesos.

El mismo autor destaca las siguientes características negativas: poseen escaso nivel tecnológico; tienen baja calidad de la producción; ausencia de normas y altos costos; falta de crédito y difícil acceso; mano de obra sin calificación; ausencia total de políticas y estrategias para el desarrollo ambiental del sector; son insuficientes los mecanismos de apoyo para el financiamiento, capacitación, y uso de tecnología limpia, y es el sector que mayormente utiliza insumos y materias primas.

En este orden de ideas, Quiñónez-Cabeza \& Parada-Gutiérrez (2013) expresan que en particular en la República del Ecuador las pymes representan el 95\% de las unidades productivas, generan el $60 \%$ del empleo, participan del $50 \%$ de la producción, tienen un amplio potencial redistributivo, refieren capacidad de generación de empleo, amplia capacidad de adaptación, flexibilidad frente a los cambios y estructuras empresariales horizontales. No obstante, predomina también una limitada tecnología y maquinaria para la fabricación de productos, poca capacitación del personal, escaso financiamiento, inadecuado equipamiento, limitada capacidad de exportación a causa del desconocimiento de procedimientos propios a las normativas de calidad exigidas en otros países, débil cadena logística con costos elevados, entre otros que restringen su competitividad. Asimismo, Borja (2015) indica que a pesar de tener un peso muy significativo sobre la sociedad económica nacional, las pymes enfrentan desafíos marcados, entre los principales se destacan el acceso a créditos productivos y la falta de organización para llegar a mercados extranjeros.

Destacan Carrión, Zula \& Castillo (2016), que una característica distintiva de muchas pymes es que tienen un origen familiar y una esencia de supervivencia. En Ecuador, solo el 5\% perdura después del tercer año de operación, generalmente por debilidades en la preparación del personal, inadecuada infraestructura e incapacidad de agregar valor a sus productos. Varios factores determinan que las pymes en un momento de crisis no logren sobrevivir, o no se estabilicen y perduren como organización: la falta de capacitación, los escasos conocimientos contables, administrativos y financieros, la deficiente planeación estratégica y el no dar importancia a la gestión del conocimiento, que podría contribuir a evitar errores en la toma de decisiones (Alfaro \& González, 2011; citado por Carrión, Zula \& Castillo, 2016).

La tendencia de trabajar en familia lleva a que se brinde poca importancia a la capacitación, a que no queden claras las funciones y obligaciones de los miembros de la organización, y a que se haga poco uso de los conocimientos administrativos y financieros y las herramientas tecnológicas para posicionarse en el sector económico al que decidan incorporarse (Madrigal et al., 2015; citado por citado por Carrión, Zula \& Castillo, 2016). Igualmente Molina (2014) destaca entre las debilidades internas de la pymes: la limitada gestión empresarial, el control de calidad y seguridad industrial deficiente, insuficiente conocimiento del mercado y del 
mercadeo, poca formación integral del recurso humano, falta de liquidez, falta de un sentido asociativo, desconocimiento de nuevas tecnologías y poco manejo de información.

Se evidencia que las pymes ecuatorianas poseen debilidades que impiden su participación en el mercado y alcanzar su competitividad debido a las falencias que poseen en los aspectos organizacionales, administrativos y contables, planeación estratégica, gestión empresarial, control de calidad, capacitación, uso de tecnologías eficientes y no contaminantes, entre otros aspectos que dificultan su buen desempeño.

\section{Las pymes y la gestión ambiental}

La gestión ambiental en las pymes comprende el conjunto de acciones y actividades que implementan las empresas dentro de sus planes y programas de conservación y protección ambiental y se convierte en una herramienta que contribuye al cumplimiento de la normativa ambiental (González, 2017).

Tal como señala León (2015) las pymes son un sector productivo vulnerable que hay que fortalecer en materia ambiental. El autor destaca que los innumerables beneficios que se derivan de la inclusión de la protección y cuidado ambiental a la gestión empresarial se pueden resumir en dos palabras, ventaja competitiva. Muchos autores definen ventaja competitiva como el valor agregado que la empresa le da a un producto o proceso para su diferenciación en el mercado, en este caso el objetivo es que dicho valor agregado sea el ambiental. Por ende, los tomadores de decisiones y hacedores de política, deben orientarse a impulsar mencionada característica.

León (2015) señala que en Ecuador de una muestra de 10.646 empresas, 2.734 empresas (26\%) tienen algún tipo de certificación ambiental, mientras que el resto (7.912) no poseen ningún tipo (74\%). Al respecto el $79 \%$ de las pequeñas, el $63 \%$ de las medianas y el $40 \%$ de las grandes empresas, no cuentan con ningún permiso ambiental (certificación de registro ambiental y licencia ambiental), a pesar de que todos los proyectos, obras o actividades a desarrollarse en el país, deberán regularizarse ambientalmente, conforme a la normativa ambiental aplicable y a la categorización ambiental nacional. Sin embargo, destaca el autor, existen tres certificaciones extras que las empresas ecuatorianas pueden obtener: una de carácter internacional, la certificación ISO 14001 y dos de carácter nacional, la certificación Punto Verde y la certificación Carbono Neutro. Al respecto, de las 2.734 empresas que poseen algún tipo de certificación, 163 $(6 \%)$ son certificadas por el ISO 14001, 87 (3\%) tienen la certificación Punto Verde y 25 (1\%) la certificación de Carbono Neutro.

Con respecto a la Norma ISO 14001, las grandes empresas son las que tienen un mayor porcentaje de esta certificación. Tal como lo indica Molina (2014), estas certificaciones son comunes en las grandes empresas por sus condiciones financieras, lo que no sucede con las pymes que las consideran complejas, costosas y difíciles de mantener.

Por otro lado, la Certificación Punto Verde, es una certificación otorgada a las empresas de producción y servicios, que hayan implementado casos de producción más limpia reduciendo la contaminación en la fuente de sus actividades y procesos, con la adopción de tecnologías limpias (MAE, 2013, citado por León, 2015). Los ejes bajo los cuales se basa la certificación son 
la gestión de desechos, gestión de papel, uso eficiente de agua, energía y combustibles; capacitación y compras responsables (MAE, 2012, citado por León, 2015). Las empresas del Ecuador todavía no cuentan con las capacidades para aplicar a una Certificación Punto Verde. Apenas el 3\% de las firmas ecuatorianas posee este reconocimiento nacional. Lo mismo sucede con la Certificación Carbono Neutro, solo el 1\% son empresas capaces de cumplir con los requisitos de esta certificación. Es decir, el 99\% de las empresas no compensa las emisiones de sus actividades económicas, situación considerada desfavorable para el planeta.

Esta certificación se otorga mediante un Sello de Carbono Neutral, como su nombre lo indica, se entrega a las empresas que llegan a un estado de carbono neutro, al estado en el que la emisión neta de gases de efecto invernadero (GEI) es cero, es decir, la cantidad de emisiones de GEI equivalen a la cantidad de GEI removidos. Las emisiones de dióxido de carbono pueden ser compensadas de varias maneras: el uso de energías renovables de alta calidad, eficiencia energética y proyectos de reforestación (Ecuador Inmediato, 2011, citado por León, 2015).

No obstante, existen empresas que están realizando algunas actividades que contribuyen a proteger el ambiente. Al respecto, Molina (2014) presenta las principales actividades medioambientales desarrolladas por las pymes en Ecuador en orden decreciente, entre las que se encuentran: ahorro de energía, reciclaje, ahorro de agua, sistemas de gestión ambiental, certificaciones ambientales, reproceso de materiales, tratamiento de residuos, monitoreo de impactos, control a proveedores y ecoeficiencia.

En este sentido, Rodríguez Becerra \& Van Hoof (s.f.), mencionan entre los aspectos de la gestión ambiental que pueden implementar las pymes los siguientes: el aumento de la eficiencia, productividad y calidad a través de una producción más limpia, enfocada hacia el manejo preventivo de la contaminación ambiental que al mismo tiempo conlleva reducciones en costos, aumentos de productividad e innovaciones de procesos y productos; la implementación de buenas prácticas operativas con acciones sencillas y de carácter preventivo como el uso eficiente del agua, energía y materia prima, las cuales se logran a través de procesos de sensibilización y capacitación de los encargados de los procesos operativos; la adopción de tecnologías más limpias que generan menos residuos; la innovación o rediseño de productos para reducir el consumo de recursos energéticos y minimizar la cantidad de material utilizado por producto o utilizar materiales reciclables y asegurar el acceso a mercados verdes a través de la gestión ambiental preventiva y el cumplimiento de los criterios de sostenibilidad ambiental, los cuales identifican los productos verdes a través de las eco-etiquetas y la certificación ISO 14.000.

Es evidente que la incorporación de la gestión ambiental en las pymes del Ecuador todavía es muy baja, los datos reportados muestran que es necesario que las pymes implementen sistemas de gestión ambiental que contribuyan a incrementar la competitividad de estas empresas al existir una relación positiva entre ambas variables. Entre las actividades de gestión ambiental implementadas por las empresas, destacan aquellas de baja complejidad desde el punto de vista tecnológico y las que requieren menores costos e inversiones para las empresas.

\section{La gestión ambiental y la competitividad en las pymes del Ecuador}


A continuación se presentan algunas experiencias relacionadas con la aplicación de la gestión ambiental en algunas pymes ubicadas en diferentes cantones del país.

En las pymes del sector curtiembre del cantón Ambato, provincia Tungurahua en Ecuador, Santana (2016) encontró que la mayor parte de los propietarios y personal (80\%) que labora en las pequeñas y medianas empresas del sector curtiembre consideran que su conocimiento sobre la normatividad ambiental que rige a estas empresas es parcial. Asimismo, Galarza \& Santana (2016) sostienen que el $40 \%$ de las pequeñas y medianas empresas tienen un cumplimiento bueno de las normas ambientales y esto les ha llevado a tener altos niveles de competitividad, como resultado del control ejercido por las autoridades ambientales y de las mejoras implementadas en los procesos productivos.

Destaca Santana (2016) que en cada una de las pymes se han tomado medidas para reducir la contaminación y por ende los impactos ambientales provocados como resultado de sus actividades; varias de estas empresas ya cuentan con una planta de tratamiento de agua, también con filtros de retención de pelo, con pozos de recuperación de residuos, se ha sustituido el uso de químicos nocivos por otros biodegradables, entre otras, sin embargo, existen aún organizaciones en las cuales por diferentes razones aún no se han podido implementar la mayoría de las acciones anteriormente mencionadas; y al mismo tiempo es evidente que en todas ellas y de acuerdo a sus posibilidades, se ha implementado al menos una práctica que aporte a mitigar el impacto ambiental.

Galarza \& Santana (2016) señalan que el 60\% de las empresas curtiembres cuenta con un sistema de gestión ambiental informal, lo cual les permite mejorar su desempeño, asumir su responsabilidad ante la contaminación causada en el ambiente y tomar los correctivos necesarios, mientras que el $40 \%$ de las pymes por el momento no tienen un sistema de gestión ambiental, pero se encuentran en el proceso de elaboración y realizando auditorías.

Mencionan las autoras que el sistema de gestión ambiental también es una de las herramientas implementadas por las empresas para mitigar la contaminación provocada por sus actividades y estos pueden ser formales como el establecido por las Normas ISO 14000, que permite la implementación de sistemas y procesos con el objetivo de mejorar el desempeño de las organizaciones, a través de la optimización de recursos y el cumplimiento de las disposiciones ambientales vigentes, e informales como por ejemplo un programa interno de reducción de desechos, o bien, los medios y métodos no documentados por los que una organización gestiona su interacción con el medio ambiente. Algunos de los beneficios que las empresas obtienen al implementar un sistema de gestión ambiental son: reducción de costos, captación de más clientes, mejorar la relación con otras empresas, mejorar el posicionamiento de la empresa en el mercado y disminuir los precios de sus productos.

Tal como lo destaca Santana (2016), varias empresas han tomado como una estrategia de competitividad el tener tecnologías más eficientes, que les permitan optimizar recursos, pero por otra parte algunas pymes han optado por alcanzar nuevos mercados tanto a nivel local como internacional. No para todas las empresas es fácil implementar ciertas estrategias de competitividad, debido a la falta de información sobre como efectuarlas o por no poseer 
suficientes recursos económicos para llevarlas a cabo; de igual forma la falta de certificaciones en base a sistemas de gestión ambiental formales es uno de los factores determinantes.

Varias pymes curtiembres han planteado y ejecutado estrategias que no solo les permitan cumplir con las políticas ambientales sino también que beneficien sus niveles competitivos; las inversiones en mejoras y cambios en diversas áreas de la empresa son esenciales para el desarrollo y permanencia de las empresas en el mercado (Galarza \& Santana, 2016).

Otra experiencia es una investigación realizada por Holguín \& Navas (2015) en pequeñas y medianas empresas que desarrollan su actividad en el Cantón Latacunga, Provincia de Cotopaxi, en las empresas que están dentro del segmento curtiembre, alimenticio e industria gráfica. Se encontró que las empresas que están dentro del segmento de la curtiembre y alimenticio tienen un mayor grado de conocimiento y aplicación de normas que respetan el medioambiente (68\% y $64 \%$ respectivamente), mientras que las imprentas tienen un mínimo conocimiento $(12 \%)$.

Los investigadores evidenciaron que a excepción de la artes gráficas, las empresas conocen sobre todas las leyes ambientales que aplican a su actividad y cuentan con un presupuesto para la investigación y desarrollo en tecnologías amigables con el ambiente, apoyan la realización de foros, seminarios o conversatorios sobre el tema con la finalidad de fomentar y adoptar leyes, normas y reglamentos, que respeten el medioambiente, mantienen convenios o alianzas con organizaciones para promover la educación ambiental en la comunidad en donde operan, siendo el segmento de la curtiembre el que obtiene el porcentaje más alto en el tema.

En la empresa alimenticia se da un cuidado especial a lo que se refiere al impacto del proceso industrial, obteniendo el 100\% de cumplimiento en la aplicación de normas que minimicen el impacto del ruido, contaminación del aire al interior de la planta, los gases emitidos, contaminación de las aguas, etc. Cuentan con un mecanismo para identificar los peligros que ocasiona en la salud y el medio ambiente, materias primas que utilizan en sus procesos, los residuos son entregados a empresas recicladoras, capacitan al personal sobre seguridad industrial y cuentan con toda la señalética requerida (Holguín \& Navas, 2015).

En otra investigación realizada por Arévalo \& Morocho (2016) en una muestra de 185 pequeñas y medianas empresas del cantón Machala, Provincia El Oro, con diez o más trabajadores, se encontró que la gran mayoría de las pymes $(62,70 \%)$ si tiene conocimiento de cuáles son las leyes medioambientales por las que se deben regir según la actividad que empleen, debido a que si esta no es aplicada correctamente serán sancionados y multados. Asimismo, un $48,11 \%$ de las pymes identifica los daños que produce al medio ambiente la actividad que realiza, determinando así que estas a su vez cumplen con los requisitos que la ley delimita para controlar que las organizaciones no sean las causantes principales de la destrucción de la naturaleza. Por otra parte, un $40 \%$ de pequeñas y medianas empresas si posee un programa de concienciación y protección del medio ambiente debido que estas están conscientes del daño que puede generar si no se toman medidas al ejecutar algún tipo de actividad. La gran mayoría de las pymes $(51,35 \%)$ no utiliza ni implementa tecnología que contrarreste el daño que ocasiona al medio ambiente, por su parte, el 87,03\% indican que no tienden a participar en programas 
medioambientales promovidos por el gobierno puesto que desconocen de los programas que el gobierno realiza.

Veintimilla (2014) en su trabajo de investigación con las pymes de la ciudad de Loja, Ecuador, reporta que el $95 \%$ de las pymes no cuenta con políticas para reducir, dentro de su tecnología actual, el consumo de energía eléctrica, agua y productos tóxicos en sus instalaciones y oficinas, por otra parte, el $91 \%$ de las pymes no se preocupa en reducir los niveles de contaminación que pueda generar sus empresas, lo que refleja la nula o poca importancia que se da a la reducción de la contaminación ambiental, por lo que se deben realizar procesos de capacitación para que adquieran un compromiso real al respecto y generen políticas en cada empresa para minimizar la contaminación ambiental.

Igualmente, el $97 \%$ de las pymes de la ciudad de Loja no se preocupa en reducir los residuos que pudiesen ensuciar las vías públicas, por lo que se hace urgente concienciar y culturizar a las pymes sobre la importancia de mantener una ciudad o comunidad limpia; asimismo, el 82\% de las pymes no se preocupa en reducir el gasto de materias primas, agua y energía, por lo que no se evidencia preocupación en el uso eficiente de los recursos, de allí que se requiere con urgencia reglamentar y definir políticas para reducir el gasto innecesario de materias primas y el consumo excesivo de los servicios básicos, y de esta manera poder hacer un uso eficiente de los recursos. También es importante destacar que el 68\% de los empleados no conoce de la existencia de una política institucional para la protección del medioambiente de la empresa, a pesar de que el $95 \%$ de los empleados señalan que reciben capacitaciones o mensajes en temas ambientales por parte de la empresa, por lo que se debe brindar mayor información con relación a la gestión ambiental y al mismo tiempo las empresas deben definir claramente sus políticas ambientales y hacerlas conocer a todo el recurso humano.

Las experiencias reportadas nos indican que existen importantes falencias a nivel de la aplicación de acciones en las pymes que contribuyan a mejorar su gestión ambiental. Es evidente que existen dificultades en la implementación de la gestión ambiental en las pymes debido la falta de conciencia ambiental de los empresarios y sus trabajadores, la falta de capacitación en procesos ambientales, la existencia obstáculos de tipo económico y la no evidencia por parte de los empresarios de la relación de la gestión ambiental con la mejora en la posición del mercado. Asimismo, destaca el alto nivel de informalidad de las pymes, el desconocimiento de la normativa ambiental vigente, la poca exigencia de los mercados locales, el poco control de las autoridades ambientales para el cumplimiento de la normativa ambiental, los elevados costos de la tecnología y el poco financiamiento de los bancos a proyectos del área ambiental.

Las empresas que han optado por implementar sistemas de gestión ambiental lo han hecho por exigencias de los mercados, por cumplimiento de normativas ambientales, reducción de costos, mejoras en su imagen ambiental y como parte de su responsabilidad social empresarial.

\section{Conclusiones}

Las principales actividades medioambientales desarrolladas por las pymes en Ecuador en orden decreciente son: ahorro de energía, reciclaje, ahorro de agua, sistemas de gestión 
ambiental, certificaciones ambientales, reproceso de materiales, tratamiento de residuos, monitoreo de impactos, control a proveedores y ecoeficiencia.

Con respecto a la Norma ISO 14001, las grandes empresas son las que tienen un mayor porcentaje de esta certificación por sus condiciones financieras, lo que no sucede con las pymes que las consideran complejas, costosas y difíciles de mantener.

Un elevado porcentaje de las pymes cuentan con un sistema de gestión ambiental informal, lo cual les permite mejorar su desempeño, asumir su responsabilidad ante la contaminación causada en el ambiente y tomar los correctivos necesarios.

Un menor porcentaje de empresas ha implementado sistemas de gestión ambiental formales para reducir la contaminación y por ende han minimizado los impactos ambientales provocados como resultado de sus actividades, además tienen un cumplimiento bueno de las normas ambientales y esto les ha llevado a tener altos niveles de competitividad, como resultado del control ejercido por las autoridades ambientales y de las mejoras implementadas en los procesos productivos.

Al implementar un sistema de gestión ambiental las empresas obtienen beneficios, tales como: reducción de costos, captación de más clientes, mejorar la relación con otras empresas, mejorar el posicionamiento de la empresa en el mercado y disminuir los precios de sus productos. Es por ello que algunas empresas han tomado como una estrategia de competitividad el tener tecnologías más eficientes, que les permitan optimizar recursos y alcanzar nuevos mercados tanto a nivel local como internacional.

Es necesario fortalecer a las pymes en materia de gestión ambiental a través de procesos de capacitación para que adquieran un compromiso real para reducir los niveles de contaminación y generen políticas en cada empresa para minimizar la contaminación ambiental. Igualmente es importante que las pymes puedan reducir el gasto de materias primas, agua y energía, de allí que se requiere con urgencia reglamentar y definir políticas para reducir el gasto innecesario de materias primas y el consumo excesivo de los servicios básicos, y de esta manera poder hacer un uso eficiente de los recursos.

\section{Agradecimiento}

El presente artículo se elaboró dentro de las actividades y patrocinio del proyecto de investigación de la Carrera Gestión Empresarial de la UMET, Sede Machala. El proyecto se denomina "Propuestas de mejoras de la competitividad interna/ empresarial de las mipymes de la Provincia de El Oro".

\section{Bibliografía}

Alcocer Calderón, S. T. y Díaz Ramos, J. P. (2016). Análisis comparativo de los resultados obtenidos luego de haber implementado el sistema de gestión de la calidad en las pymes dedicadas al comercio al por mayor, ubicadas en la ciudad de Quito, en el período 20092013. Tesis de grado para la obtención del grado de Magister en Sistemas de Gestión 
Integrados. Escuela Politécnica Nacional. 98 p. Recuperado de http://bibdigital.epn.edu.ec/bitstream/15000/16570/1/CD-7230.pdf

Arévalo Herrera, G. S. y Morocho Guambo, D. P. (2016). Análisis de la Responsabilidad Social Empresarial en las pequeñas y mediana empresas de la ciudad de Machala. Trabajo de titulación de Economista con mención en Gestión Empresarial. Universidad Técnica de Machala. 67 p. Recuperado de http://repositorio.utmachala.edu.ec/handle/48000/9496

Bercovich, N. y López, A. (2005). Políticas para mejorar la gestión ambiental en las pymes argentinas y promover su oferta de bienes y servicios ambientales. CEPAL. Serie medio ambiente y desarrollo, (96). 66 p. Chile. Recuperado de http://repositorio.cepal.org/bitstream/handle/11362/5632/1/S05293_es.pdf

Borja Marcial, B. G. (2015). Grado de aplicación de los sistemas de gestión que utilizan las normas ISO y OHSAS en las pymes de la industria manufacturera del distrito metropolitano de Quito - caso: proyecto de investigación k1310 subsector ciiu c18 impresión y reproducción de grabaciones. Trabajo de titulación de grado para la obtención del título de Ingeniería Comercial. Pontificia Universidad Católica del Ecuador. 110 p. Recuperado de http://repositorio.puce.edu.ec/handle/22000/10164

Carrión, L.; Zula, J. y Castillo, L. (2016). Análisis del modelo de gestión en pequeñas y medianas empresas y su aplicación en la industria del catering en Ecuador. 82-102. Recuperado de https://www.uv.mx/iiesca/files/2016/11/09CA201601.pdf

Galarza Galarza, J. C. y Santana Arellano, A. del P. (2016). Normas ambientales y competitividad en las Pymes del sector Curtiembre de Ambato-Ecuador. Revista Digital de Medio Ambiente "Ojeando la agenda", (4), 14-30, noviembre. Recuperado de: https://mirevistadigital.files.wordpress.com/2016/11/art-cumplimiento-de-las-normas.pdf

González Ordóñez, A. I. (2017). La gestión ambiental en la competitividad de las pymes. Revista Científica Agroecosistemas, 5 (1), 60-70. Recuperado de http://aes.ucf.edu.cu/index.php/aes/index

Holguín Varea, M. R. y Navas Salazar, R. S. (2015). Investigación sobre la aplicación de prácticas de responsabilidad social en las pymes del Cantón Latacunga y la elaboración de un manual práctico. Tesis presentada como requisito previo a la obtención del título de Magíster en Gestión de Empresas. Universidad de las Fuerzas Armadas. 159 p. Recuperado de http://repositorio.espe.edu.ec/xmlui/handle/21000/9418

León Bolaños, N. V. (2015). Propuesta de instrumentos de política pública que promuevan la protección y cuidado ambiental en la gestión empresarial del Ecuador. Tesis de grado para la obtención del grado de Economista. Pontificia Universidad Católica del Ecuador. 135 p. Recuperado de http://repositorio.puce.edu.ec/bitstream/handle/22000/10481/Disertaci\%C3\%B3n\%20co mpleta.pdf? sequence $=1 \&$ isAllowed $=y$ 
Molina Galarza.; M.A. (2014). Propuesta de un modelo de Responsabilidad Social Empresarial para PYMES con elementos de la Norma ISO 26000. Tesis para la obtención del título de Máster en Administración de Empresas. Universidad del Azuay. 132.p. Recuperado de http://dspace.uazuay.edu.ec/bitstream/datos/4075/1/10672.pdf

Ojeda López, R. N. y Mul Encalada, J. (2015). La gestión ambiental y su relación con la competitividad: un estudio aplicado en las micro, pequeñas y medianas empresas del estado de Yucatán, México. Revista Tec Empresarial, 9 (2), 41-49, agosto-octubre. Recuperado de https://dialnet.unirioja.es/descarga/articulo/5181427.pdf

Quiñónez-Cabeza, M. R. y Parada-Gutiérrez, C. O. (2013). Análisis de la gestión competitiva de las pequeñas y medianas empresas comerciales de Esmeraldas, República del Ecuador. Anuario Facultad de Ciencias Económicas y Empresariales, 4, 90-100. Recuperado de revistas.uo.edu.cu/index.php/aeco/article/download/627/602

Rodríguez Becerra, M. y Van Hoof, B. (s.f.). Para que la pyme sea más competitiva se requiere una gestión ambiental preventiva. 8 p. Recuperado de www.manuelrodriguezbecerra.org/bajar/pymes.pdf

Saavedra García, M., Milla Toro, S. y Tapia Sánchez, B. (2013). Determinación de la competitividad de la Pyme en el nivel micro: el caso del Distrito Federal, México. Revista FIR, FAEDPYME International Review, 2 (4), 38-52 julio-diciembre. Recuperado de http://faedpyme.ojs.upct.es/index.php/revista1/article/view/38

Santana Arellano, A. (2016). El cumplimiento de las normas ambientales y su relación con la competitividad de las Pymes del sector curtimbre del cantón Ambato, 2016. Universidad Técnica de Ambato. Facultad de Contabilidad y Auditoría. Carrera de Economía. Proyecto de Investigación previo la obtención del título de Economista. 124 p. Recuperado de http://repositorio.uta.edu.ec/jspui/handle/123456789/23945

Van Hoof, B. (2005). Políticas e instrumentos para mejorar la gestión ambiental de las pymes en Colombia y promover su oferta en materia de bienes y servicios ambientales. CEPAL. Serie medio ambiente y desarrollo, (94). 77 p. Chile. Recuperado de http://repositorio.cepal.org/bitstream/handle/11362/5634/1/S05291_es.pdf

Veintimilla Carrión, M. A. (2014). Conocimiento e inserción de la responsabilidad social empresarial en las pymes de la ciudad de Loja período 2013-2014. Universidad Técnica Particular de Loja. Titulación de Ingeniero en Administración de Empresas. 110 p. Recuperado de http://dspace.utpl.edu.ec/handle/123456789/10677 